\title{
Irisin Enhances Angiogenesis of Mesenchymal Stem Cells to Promote Cardiac Function in Myocardial Infarction via PI3k/Akt Activation
}

\author{
Fan Yang ${ }^{1,2}$, Zhi Wang ${ }^{3}$, Bing $\mathrm{Li}^{1,2}$, Youfu He ${ }^{1,2}$, Fawang $\mathrm{Du}^{1,2}$, Shui Tian $^{1,2}$, Yu Zhang ${ }^{4}$, Yongyao Yang ${ }^{1,2}$ \\ ${ }^{1}$ Department of Cardiology, Guizhou Provincial People's Hospital, Guiyang, China \\ ${ }^{2}$ Department of Cardiology, Guizhou University People's Hospital, Guiyang, China \\ ${ }^{3}$ Qingdao Municipal Hospital (Group), Qingdao, China \\ ${ }^{4}$ Department of Cardiology, Xixiu District People's Hospital, Anshun, China
}

Background and Objectives: With the growing incidence of acute myocardial infarction (MI), angiogenesis is vital for cardiac function post-MI. The role of bone marrow mesenchymal stem cells (BMSCs) in angiogenesis has been previously confirmed. Irisin is considered a potential vector for angiogenesis. The objective of the present study was to investigate the potential role of irisin in the angiogenesis of BMSCs.

Methods and Results: In vivo, irisin-treated BMSCs (BMSCs + irisin) were transplanted into an MI mouse model. On day 28 post-MI, blood vessel markers were detected, and cardiac function and infarct areas of mice were evaluated. In vitro, paracrine effects were assessed by examining tube formation in human umbilical vein endothelial cells (HUVECs) co-cultured with the BMSCs + irisin supernatant. The scratch wound-healing assay was performed to evaluate HUVEC migration. Western blotting was performed to determine PI3k/Akt pathway activation in the BMSCs+irisin group. Transplantation of BMSCs + irisin promoted greater angiogenesis, resulting in better cardiac function in the MI mouse model than in controls. In the BMSC+irisin group, HUVECs demonstrated enhanced tube formation and migration. Activation of the PI3k/Akt pathway was found to be involved in mediating the role of irisin in the angiogenesis of BMSCs.

Conclusions: In cardiovascular diseases such as MI, irisin administration can enhance angiogenesis of BMSCs and promote cardiac function via the PI3k/Akt pathway, optimizing the therapeutic effect based on BMSCs transplantation.

Keywords: Irisin, Angiogenesis, Mesenchymal stem cells, PI3k/Akt pathway

Received: January 6, 2021, Revised: May 25, 2021,

Accepted: July 1, 2021, Published online: August 31, 2021

Correspondence to Yongyao Yang

Department of Cardiology, Guizhou Provincial People's Hospital,

NO. 83 Zhongshan East Road, Guiyang 550002, China

Tel: +86-851-8592-5127, Fax: +86-851-8592-5127

E-mail: 729142921@qq.com

(c) This is an open-access article distributed under the terms of the Creative Commons Attribution Non-Commercial License (http://creativecommons.org/ licenses/by-nc/4.0/), which permits unrestricted non-commercial use, distribution, and reproduction in any medium, provided the original work is properly cited.

Copyright (c) 2021 by the Korean Society for Stem Cell Research

\section{Introduction}

In acute myocardial infarction (MI), atherosclerotic vascular occlusions trigger pathological injury owing to myocardial ischemia and hypoxia. Angiogenesis and vascular remodeling play essential roles in affording protection against myocardial ischemia (1). Notably, angiogenesis primarily decelerates the development of heart failure (2). Percutaneous coronary intervention (PCI) and coronary artery bypass grafting have failed to rescue microvascular injury and reportedly result in PCI-related microvascular damage (3). Thus, new medical approaches have revealed a critical role for angiogenesis against progressive cardiac 
deterioration during cardiovascular diseases. In previous studies, as well as in our present study, human bone marrow mesenchymal stem cells (BMSCs) improved angiogenesis through a paracrine effect, primarily rescuing cardiac function in the MI mouse model (4).

Irisin, a new multifunctional myokine related to metabolic factors, is predominantly released from skeletal muscle and myocardium. Irisin is known to be involved in cell physiology and pathology, reportedly associated with the browning of white adipocytes and increased energy expenditure (5). Irisin is cleaved from fibronectin type-III domain-containing protein 5 (FNDC5) and released into the circulation, which is reportedly involved in energy metabolism in skeletal muscle, adipose tissue, and pancreatic islets (6). In a transverse aortic constriction (TAC)-induced cardiac hypertrophy mouse model, irisin reportedly alleviated cardiac fibrosis and hypertrophy, thus improving cardiac function through 5'-adenosine monophosphate-activated protein kinase (AMPK)-mammalian target of rapamycin (mTOR) signaling (7). Similarly, irisin attenuates cardiac fibrosis in TAC-induced hypertrophy by increasing autophagy (8). Furthermore, irisin is involved in the angiogenesis of human umbilical vein endothelial cells (HUVECs), thus protecting cardiac function after MI (9, 10). Recent studies have reported that irisin regulates the crosstalk between adipose tissue and muscle via paracrine signaling (10). However, the role of irisin in the paracrine signaling of BMSCs needs to be elucidated. In the present study, we hypothesized that irisin could enhance the paracrine effect of BMSCs on angiogenesis to improve cardiac function post-MI injury.

\section{Materials and Methods}

\section{Animal experiment}

All animal procedures were conducted in accordance with the guidelines of the Animal Use Committee of the Guizhou Provincial People's Hospital. To establish the MI mouse model, the left anterior descending coronary artery of 8 12-week old male C57BL/6J mice (Guizhou Medical University) was ligated as previously described (11). BMSCs with/without irisin treatment $\left(1.5 \times 10^{5}\right.$ cells suspended in $20 \mu 1$ Dulbecco's Modified Eagle Medium [DMEM] per mouse) were immediately transplanted into the border zone at four different sites. The DMEM group received DMEM without BMSCs (mice received an equivalent volume of DMEM).

\section{Human BMSCs isolation and culture}

Based on a previously reported protocol (11), human
BMSCs were isolated and cultured from patients who underwent hip replacement upon receipt of informed consent and approval by the Human Ethics Committee of Guizhou Provincial People's Hospital.

BMSCs at $4-8$ passage were treated with irisin $(200 \mathrm{ng} / \mathrm{ml}$; Phoenix Pharmaceuticals, USA) and LY294002 (50 $\mu \mathrm{M}$; Selleck Chemicals, USA), as previously reported (10), for $24 \mathrm{~h}$ under normoxic conditions. Dimethyl sulfoxide (DMSO), the solvent employed for irisin, was used as a control.

\section{Immunostaining}

Twenty-eight days post-MI, mice were sacrificed, and their hearts were harvested and embedded in O.C.T. compound (Sakura Finetek, USA). Heart tissue sections (7- $\mu \mathrm{m}$ thick) were fixed using $4 \%$ paraformaldehyde. After permeabilization with $0.2 \%$ Triton, tissue slices were blocked with $3 \%$ bovine serum albumin (BSA) and incubated with primary antibody at $4^{\circ} \mathrm{C}$ overnight. Sections were incubated with the secondary antibody at room temperature for $1 \mathrm{~h}$ and then observed using a fluorescence microscope (Leica, Germany). The vascular density at border areas was calculated as the positive number of vascular markers per area for one high-power field $\left(34.68 \mu \mathrm{m}^{2}\right)$, with measurements performed in at least five random high-power fields for each section.

\section{Sirius Red staining}

Twenty-eight days post-MI, mice were euthanized, and hearts were harvested and embedded in paraffin. After sectioning (3- $\mu \mathrm{m}$ thick sections), the cardiac tissue was stained using Sirius Red (Solaribio, Beijing, China), according to the manufacturer's instructions. Infarct areas were measured using Image-Pro software (Media Cybernetics, USA) and assessed according to the sum of the endocardial and epicardial length of the infarct zone in proportion to the sum of the endocardial and epicardial length of the entire left ventricle.

\section{Tube formation assay}

Tube formation was assessed using HUVECs, co-cultured with the supernatant of BMSCs treated with irisin, LY294002, irisin+LY294002, or control. HUVECs were seeded at a density of $5 \times 10^{4}$ cells per well and co-cultured with the BMSC supernatant. Tube formation images were acquired $4 \mathrm{~h}$ after transplantation, and the number of branch points was quantified using the Image-Pro software.

\section{Scratch wound-healing assay}

The wound-healing assay was performed as previously 
described (10). Briefly, HUVECs were cultured in a 6-well plate $\left(1 \times 10^{6}\right.$ cells/well) for $48 \mathrm{~h}$ under normal conditions. After scratching the surface with a sterile $10-\mu 1$ pipette tip, HUVECs were washed twice and cultured in DMEM (containing $1 \%$ fetal bovine serum [FBS]) for $24 \mathrm{~h}$, containing an equal volume of BMSC supernatant treated with irisin, LY294002, irisin + LY294002, or an equivalent volume of DMSO. The wound areas were photographed with an inverted microscope (Leica, Germany), and cell migration was calculated as the average wound area in five random fields, compared with the zero-time point, using Image-Pro software.

\section{Cell apoptosis assay}

Following treatment with irisin for $24 \mathrm{~h}$ under normal conditions, BMSCs were cultured in DMEM with glucose and $\mathrm{FBS}$ deprivation under hypoxia $\left(95 \%\right.$ air $/ 5 \% \mathrm{CO}_{2}$, at $37^{\circ} \mathrm{C}$ ) for $24 \mathrm{~h}$.

\section{TUNEL staining assay}

Apoptosis was detected using the TUNEL Apoptosis Assay Kit (Beyotime Biotechnology, Shanghai, China) according to the manufacturer's instructions. After fixation with $4 \%$ paraformaldehyde for $30 \mathrm{~min}$, BMSCs were permeabilized with $0.2 \%$ Triton for $5 \mathrm{~min}$ at room temperature, followed by incubation with TUNEL reaction reagent for $1 \mathrm{~h}$ at $37^{\circ} \mathrm{C}$ in the dark. Images were captured using a fluorescence microscope. Apoptotic cells were calculated as TUNEL-positive cells in proportion to the total number of cells.

\section{Western blotting}

Western blotting was performed to detect protein levels of p-PI3K, PI3K, p-Akt, and Akt. In brief, the proteins were obtained from cells using RIPA lysis buffer on ice, followed by centrifugation for clarification. Western blotting was performed as previously described (12).

\section{Echocardiography}

Echocardiography was performed to assess cardiac function on day 28 post-MI. After isoflurane inhalation, the mice were anesthetized and bound. Then, two-dimensional images were obtained using a Vevo 2100 system (VisualSonics, Inc., Toronto, Canada).

\section{Statistical analysis}

All experiments were independently performed at least three times, and data values are presented as the mean \pm standard error of the mean. Student's t test was used to compare any two groups, and more than three groups were compared using one-way analysis of variance. ${ }^{*} \mathrm{p}<0.05$ was deemed statistically significant.

\section{Results}

\section{Irisin enhanced the protection of BMSCs in the MI mouse model}

The therapeutic efficacy of BMSCs in ischemic heart disease has been clarified in several animal studies and clinical trials $(13,14)$. To confirm the protective role of irisin toward BMSCs, we treated BMSCs with irisin (200 $\mathrm{ng} / \mathrm{ml}$ ) for $24 \mathrm{~h}$ under normoxic conditions (BMSCs + iri$\sin )$. DMSO, the solvent used for irisin, was used as the control (BMSCs+Con). In the MI mouse model, we transplanted BMSCs treated with irisin into the border zone immediately after MI surgery. Twenty-eight days post-MI, improved heart function was noted, with an increased ejection fraction (EF) and fractional shortening (FS), using two-dimensional echocardiography in the BMSCs+ irisin group when compared with the BMSCs +Con and DMEM groups (Fig. 1a and 1b). Moreover, the infarct areas were examined by Sirius Red staining, presenting reduced infarct areas in the BMSCs + irisin group when compared with the BMSCs+Con and DMEM groups (Fig. 1c and 1d). These results revealed that irisin protected BMSCs in response to ischemic insults in vivo.

\section{Irisin regulated angiogenesis of BMSCs in vivo}

The angiogenic efficacy of BMSCs mediated via paracrine signaling has been reported (4). Additionally, the role of irisin in angiogenesis was previously verified (9, 10). For further evaluation, we investigated the ability of irisin-regulated BMSC paracrine signaling to induce angiogenesis. On day 28 post-MI modeling, alpha-smooth muscle actin ( $\alpha$-SMA), a marker of vascular smooth muscle cells, was significantly elevated in the BMSCs + irisin group when compared with the BMSCs+Con and DMEM groups (Fig. 2a). Similarly, vascular endothelial cells markers, including von Willebrand factor (vWF) and CD31, were considerably enhanced in the BMSCs + irisin group when compared with the BMSCs+Con and DMEM groups (Fig. $2 \mathrm{~b}$ and $2 \mathrm{c}$ ). Interestingly, these vascular markers were marginally increased in the BMSCs+Con group when compared with the DMEM group. Blood vessels in the border zone were quantified (Fig. 2d), indicating that irisin further promoted the angiogenic efficiency in BMSCs, despite the moderate efficiency of BMSCs in angiogenesis. 
(a)

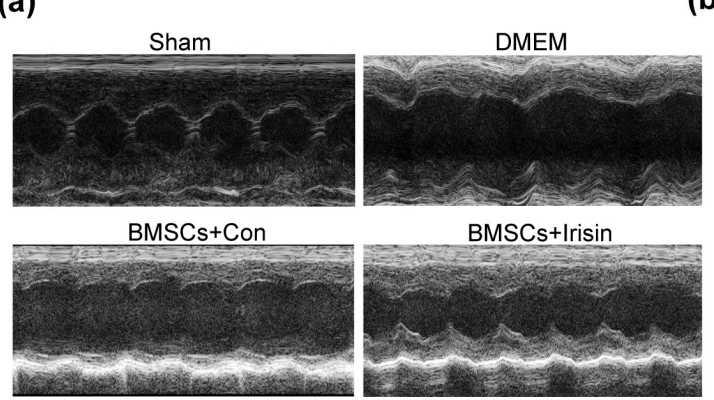

(c)
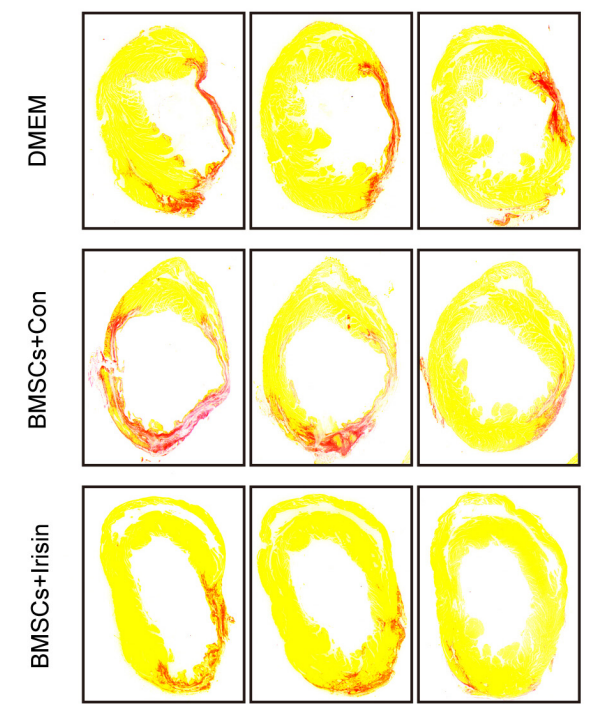

(b)
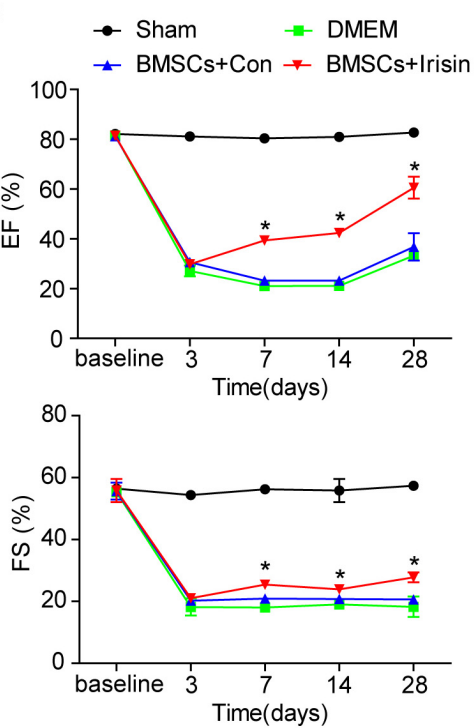

(d)

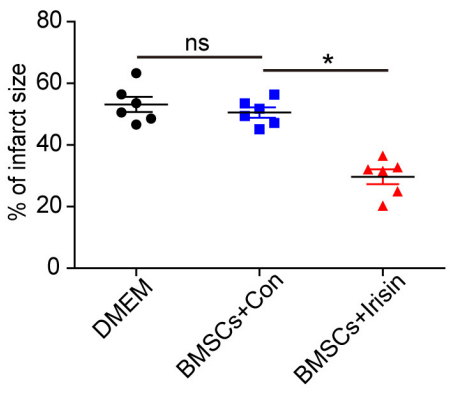

Fig. 1. Irisin-treated BMSCs rescued the cardiac function in MI mouse in vivo. (a) Representative images of echocardiography showing the improved cardiac function in BMSCs treated with irisin. (b) The ejection fraction (EF) and fractional shortening $(\mathrm{FS})$ are gradually recovered in the BMSCs + irisin group when compared with other groups $(\mathrm{n}=8$ for Sham, 5 for DMEM, 6 for BMSCs+ irisin and BMSCs+Con). (c) The infarct area was examined by Sirius Red staining 28 days post-Ml $(\mathrm{n}=6$ for every group). (d) The infarct size in hearts was quantified using Image-Pro. All data were measured as mean \pm standard error of the mean (SEM). ${ }^{*} p<0.05$. BMSCs, bone marrow mesenchymal stem cells; Ml, myocardial infarction; DMEM, Dulbecco's Modified Eagle Medium.

\section{Irisin mediated the paracrine effects of BMSCs by} primarily regulating the PI3K/Akt pathway

Previous studies have shown that the paracrine effects of BMSCs influence cellular fate $(4,15)$. To determine the role of irisin in mediating paracrine effects of BMSCs, conditioned media of BMSCs, with or without irisin, were collected; then, tube formation of HUVECs was assessed using the Matrigel assay, with cell migration examined using a wound-healing assay. In the BMSCs + irisin group, tube formation (Fig. 3a and 3c) and migration (Fig. 3b and 3d) of HUVECs were significantly increased when compared with the BMSCs + Con and irisin alone groups, but not in control alone; no difference was observed between the BMSCs+Con group and irisin alone group. The results indicated that irisin enhanced the paracrine effects of BMSCs, consistent with a previous study (16).

In MI, the PI3k/Akt pathway is deemed the primary signal transduction pathway associated with cardioprotection (17). In a cardiotoxicity model, irisin was found to be involved in cardiomyocyte apoptosis via Akt activation (6). Herein, we sought to monitor the level of PI3k/Akt in irisin-regulated BMSC paracrine signaling. In the present study, p-PI3k/PI3k and p-Akt/Akt were increased in irisin-mediated BMSCs when compared with the control group, with no differences in the p-ERK1/2/ERK1/2, $\mathrm{p}-\mathrm{P} 38 / \mathrm{P} 38$, and $\mathrm{p}-\mathrm{JNK} / \mathrm{JNK}$ pathways, revealing that $\mathrm{PI} 3 \mathrm{k} /$ Akt was involved in irisin-regulated BMSC paracrine signaling (Fig. 3e). Furthermore, inhibition of the PI3k/ Akt pathway (LY294002) was performed to suppress irisin-induced tube formation (Fig. 4a and 4b). In the wound-healing assay, the increased HUVEC migration, regulated by irisin-treated BMSCs, was blocked by LY294002 (Fig. 4c and 4d). However, a slight increased effect of tube formation and HUVEC migration by irisin despite LY294002 administration, suggesting that alternate signaling pathways are involved in irisin-regulated BMSC paracrine signaling (Fig. $4 \mathrm{c}$ and $4 \mathrm{~d}$ ). These data revealed that irisin promoted BMSC-mediated paracrine signaling via the PI3k/Akt pathway. 
(a)
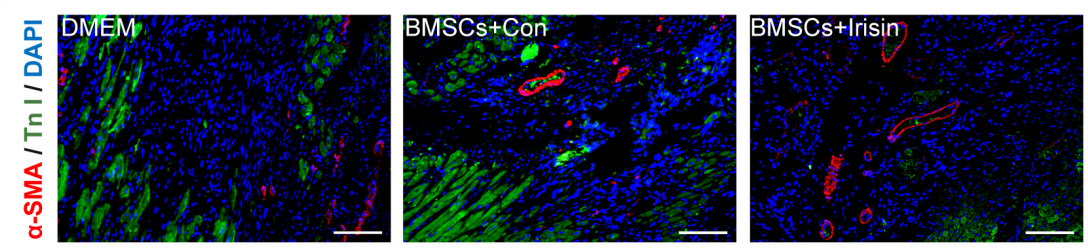

(b)
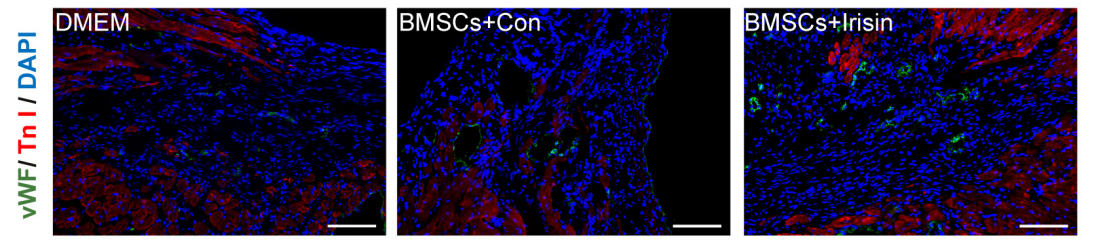

(c)
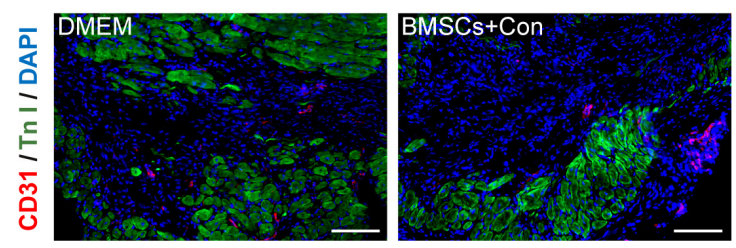

(d)
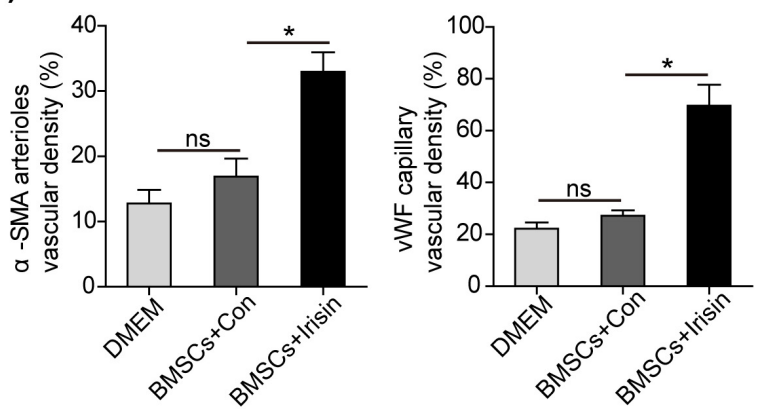

\section{Discussion}

In the present study, we observed that the irisin administration increased the angiogenesis of BMSCs in the border zone to protect cardiac function post-MI in vivo. Consistent with this phenomenon in vivo, irisin treatment promoted the paracrine efficacy of BMSCs in vitro. Mechanistically, we observed that irisin activated the PI3k/Akt signaling pathway, primarily improving the paracrine efficiency of BMSCs. Combined with these data, it was speculated that irisin could be a potential novel therapeutic agent for BMSC transplantation in tissue engineering and regenerative medicine for cardiovascular diseases.

Mesenchymal stem cells (MSCs) are a promising therapeutic option for immune-mediated diseases, cardiovascular diseases, diabetes, and cancer $(13,18,19)$. The para-
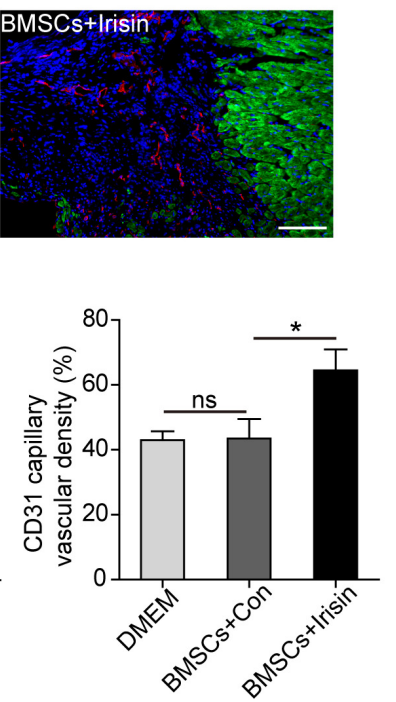

Fig. 2. Irisin-treated BMSCs enhance angiogenesis. $(\mathrm{a} \sim \mathrm{c})$ Representative images of vascular markers including $\alpha$-SMA, vWF and CD31 were captured in the border zone of irisintreated BMSCs, control, and DMEM groups. (d) The vascular density was measured in at least 5 high-power fields per section ( $\mathrm{n}=8$ for DMEM, 7 for BMSCs+irisin and BMSCs+ Con). Scale bar, $100 \mu \mathrm{m}$. Data are presented as mean \pm standard error of the mean (SEM). ${ }^{*} p<0.05$. BMSCs, bone marrow mesenchymal stem cells; DMEM, Dulbecco's Modified Eagle Medium; $\alpha$-SMA, alpha-smooth muscle actin; VWF, von Willebrand factor.

crine effect of MSCs is reportedly considered the principal mechanism underlying MSC-based therapy, including exosomes and other paracrine factors such as basic fibroblast growth factor (bFGF) and vascular endothelial growth factor (VEGF) $(13,20)$. Based on the paracrine action, MSCs enhance angiogenesis via HIF- $1 \alpha$, miR-200b, and the leptin signaling pathway (17). Similarly, the paracrine function was observed in the present study, as revealed by the Matrigel assay.

In skeletal muscles, irisin is a myokine cleaved from FNDC5, especially after exercise. The role of irisin is multifunctional, with activities such as anti-inflammation, neuroprotection, bone formation, and metabolic regulation $(21,22)$. As a hormone secreted into circulation, irisin has been shown to afford protection against cardiovascular injury, reduce cardiac fibrosis, and improve cardiac function $(23,24)$. Moreover, irisin is considered crosstalk in muscle 
(a)

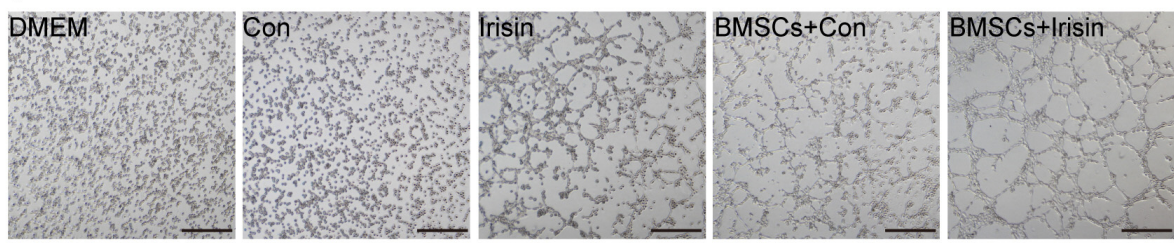

(b)
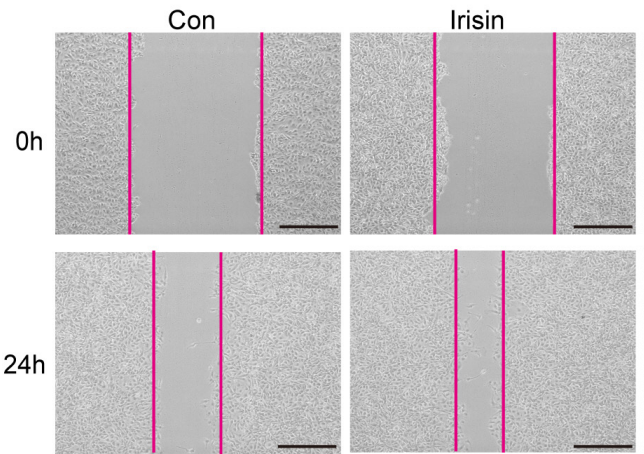

(e)

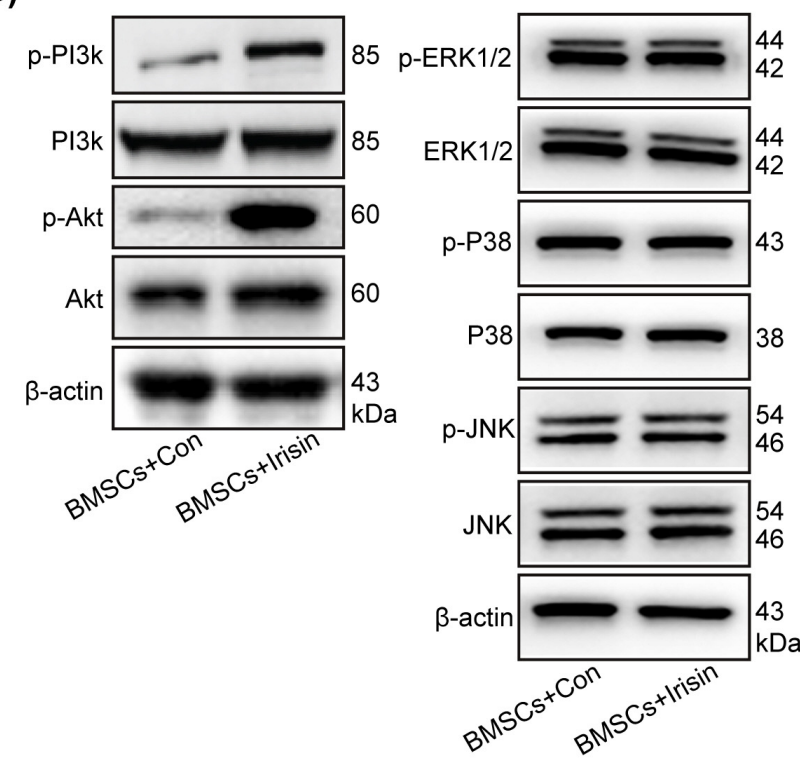

BMSCs+Con
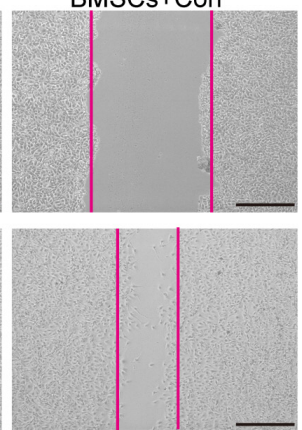

(c)

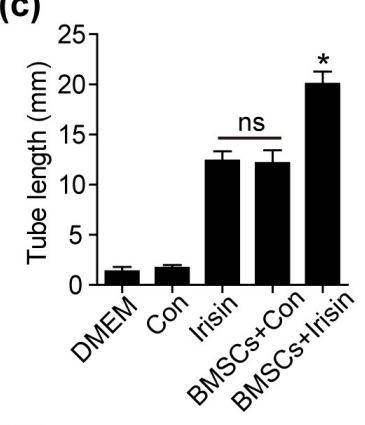

(d)
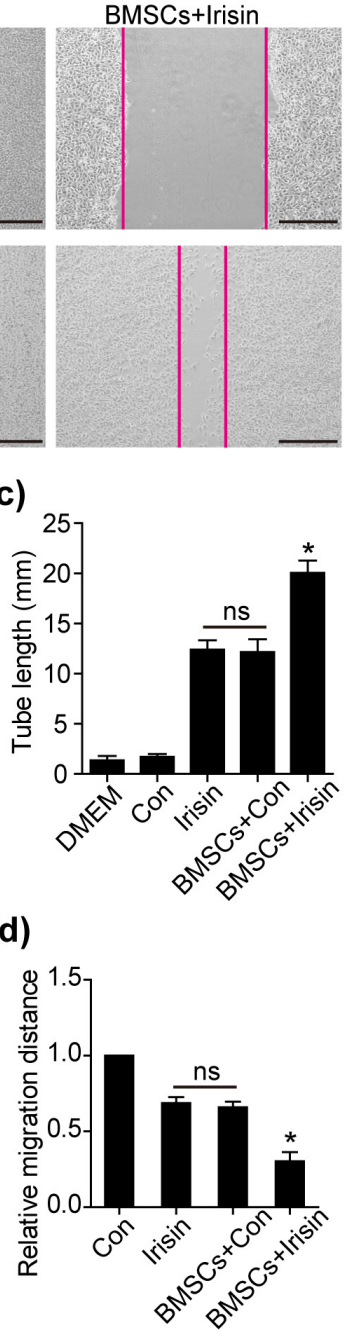

Fig. 3. Irisin promotes the paracrine efficacy of BMSCs through the PI3K/AKT pathway. (a) Conditioned media of equivalent cell numbers $\left(1 \times 10^{6}\right.$ cells $)$ were collected for irisin-treated BMSCs and control groups. By employing HUVECs, tube formation was assessed using the supernatant of BMSCs treated with irisin and control, irisin alone, and control alone. Scale bar, $50 \mu \mathrm{m}$. (b) The wound-healing assay was performed to demonstrate HUVEC migration using the above conditioned media, respectively. Scale bar, $243.2 \mu \mathrm{m}$. (c) The number of branch points was quantified by Image-Pro software and is shown in bar graphs $(n=3$ separate studies). (d) Quantification of HUVEC migration by Image-Pro software $(n=3)$. (e) Western blot analyses of pPI3K, PI3K, p-AKT, AKT, p-ERK1/2, ERK1/2, p-P38, P38, $\mathrm{p}-\mathrm{JNK}$, and JNK pathway were detected in irisin-treated BMSCs and control groups. All data were measured as mean \pm standard error of the mean (SEM). ${ }^{*} p<0.05$. BMSCs, bone marrow mesenchymal stem cells; HUVECs, human umbilical vein endothelial cells. -adipose-bone-neuron connectivity mediated by paracrine or endocrine activity $(25,26)$. However, the functional role of irisin in mediating the paracrine effects of MSCs remains elusive. In the present study, we further demonstrated that irisin treatment enhanced the paracrine function of MSCs. Despite irisin functions discovered in several previous studies, our data revealed a new aspect of irisin-related paracrine effects in MSCs.

The therapeutic potential of BMSCs has been reported in several studies (27). However, the lower viability, inefficient homing, and migration remain major hurdles in the development of BMSC-mediated treatment strategies; therefore, numerous studies have attempted to improve the ability of BMSCs, including genetic modification and target tissue modification $(28,29)$. Although we failed to demonstrate the cardioprotection afforded by BMSCs, irisin showed a trend toward improvement when compared with DMEM. Meanwhile, we observed that irisin enhanced not only the angiogenesis of BMSCs but also their survival (Supplementary Fig. S1), which was consistent with a previous study demonstrating irisin-mediated improvements on BMSCs in an MI model (16). 
(a)
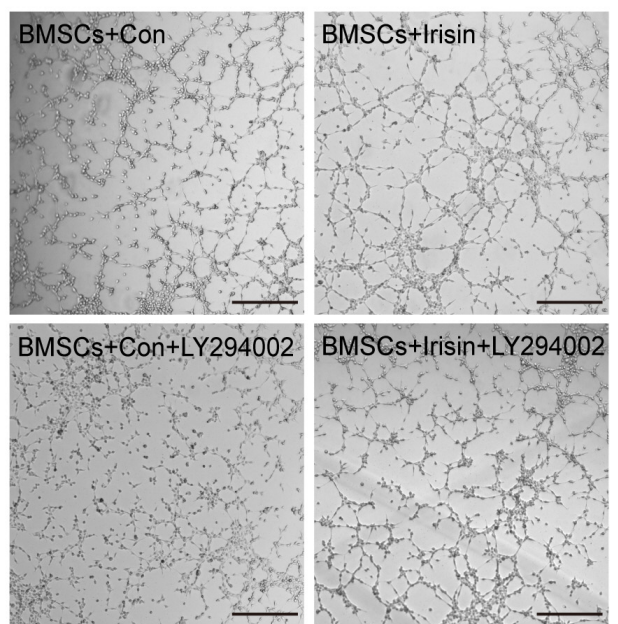

(c)
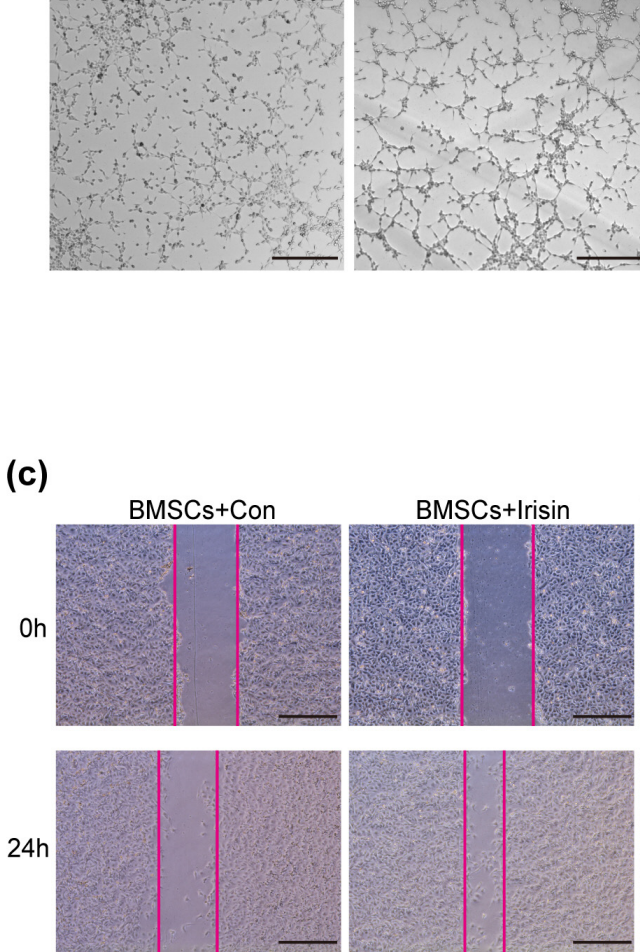

(b)

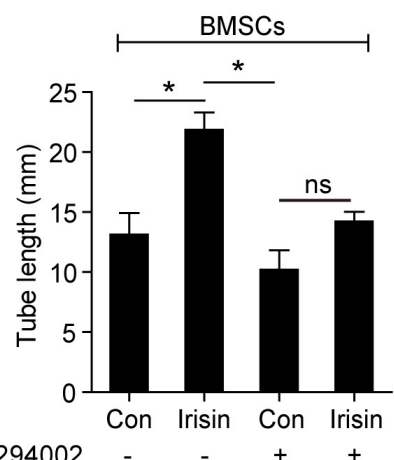

(d)

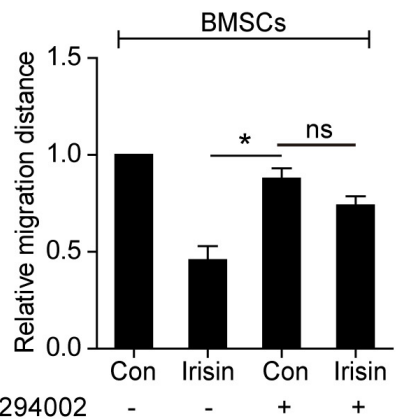

BMSCs+Con+LY294002 BMSCs+|risin+LY294002
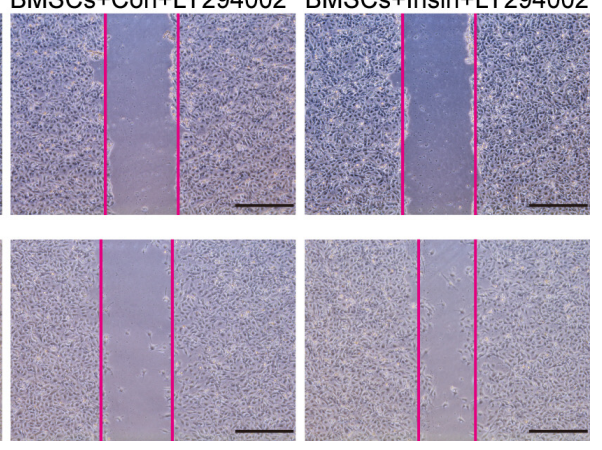

Fig. 4. The PI3K/AKT pathway is required to mediate the paracrine efficacy of BMSCs. (a) Representative images of tube formation were analyzed using HUVECs co-cultured with the supernatant of BMSCs treated with irisin, LY294002, irisin+ LY294002, or control, respectively. Scale bar, $50 \mu \mathrm{m}$. (b) The number of branch points in the above groups was quantified by Image-Pro software and is shown in bar graphs $(n=3)$. (c) The wound-healing assay assessed HUVEC migration using the above conditioned media, respectively. Scale bar, $243.2 \mu \mathrm{m}$. (d) Quantification of HUVEC migration $(n=3)$ by Image-Pro software. Data are presented as mean \pm standard error of the mean $(\mathrm{SEM}) .{ }^{*} \mathrm{p}<0.05$. BMSCs, bone marrow mesenchymal stem cells; HUVECs, human umbilical vein endothelial cells.
The mechanism via which irisin exerts its paracrine function remains unclear. Mitogen-activated protein kinases (MAPKs), as a major signaling pathway of irisin, play a critical role in cellular mitosis, differentiation, metabolism, and apoptosis (5). However, we failed to determine the influence of irisin on the ERK1/2, P38, and JNK pathways in BMSCs. As irisin regulates neural differentiation and endothelial cell proliferation via the ERK1/2 pathway and mediates browning of white adipocytes via the P38 pathway (30), we speculated that irisin mainly activated these pathways during cell proliferation; however, we detected that irisin had no impact on BMSC proliferation as examined by the Cell Counting Kit 8 (CCK-8) assay (data not shown).

The regulation of the PI3k/Akt signaling pathway is involved in a large spectrum of cellular fates, including growth, proliferation, metabolism, and survival (31). Reportedly, improved MSC survival and paracrine signaling have been associated with the activation of the PI3k/Akt pathway in a mixture of moringin and cannabidiol-induced apoptosis and heat stress-induced skin injury models $(32,33)$. Moreover, inhibition of the PI3k/Akt pathway could trigger cancer stem cell death and alleviate ovarian cancer (34). However, irisin regulation via the PI3k/Akt pathway needs to be comprehensively investigated. Zhang et al. (35) have observed that irisin modulated apoptosis of pancreatic cancer cells by inactivating the PI3K/AKT pathway. Liu et al. (36) have reported that irisin inhibits doxorubicin-induced apoptosis in pancreatic cancer. Meanwhile, in liver cancer, irisin increased cell proliferation and invasion via activation of the PI3K/ AKT pathway (37). In a mouse model of type 2 diabetes, 
Liu et al. (38) have demonstrated that irisin improves insulin resistance and insulin sensitivity via PI3K/AKT pathway activity. It has been suggested that irisin may bind to different cellular receptors to activate or inhibit the PI3K/AKT pathway in diverse cells. Therefore, our study determined the effect of irisin on the PI3K/AKT pathway following MSC transplantation in an MI mouse model. PI3K kinases are a family of enzymes that constitute three classes, Class I (p85/p110 and p101/p120), Class II (PI3KC2 $\alpha$, PI3KC2 $\beta$, and PI3KC2 $\gamma$ ), and Class III (VPS34), and p85 is the primary and canonical subunit of PI3K (39). We observed that the PI3K/AKT pathway was activated following irisin administration using the PI3K p85 antibody. However, irisin demonstrated marginal paracrine effects following administration of a PI3k/Akt pathway inhibitor, indicating other minor signaling pathways are involved in mediating the effects of irisin, such as PGC-1 $\alpha$ and IGF-1 (40).

In conclusion, in the present study, we demonstrated that irisin promoted the angiogenesis and paracrine effects of BMSCs to improve cardiac function in MI model mice through the PI3k/Akt pathway. Accordingly, we propose a novel optimized MSC strategy for regenerative medicine.

\section{Acknowledgments}

The present work was supported by National Natural Science Foundation of China (No. 81960083), the Science and Technology Fund of Guizhou Provincial Health Commission (gzwjkj2019-1-094), Post-subsidy funds of the National Natural Science Foundation of China (GPPH-NSFC-2019-22), Guizhou Province Science and Technology Platform and Talent Team Planning Project (Qiankehe Platform Talent 2017-5405), and Anshun Science and Technology Plan Project (asks 2019-07).

\section{Potential Conflict of Interest}

The authors have no conflicting financial interest.

\section{Supplementary Materials}

Supplementary data including one figure can be found with this article online at https://doi.org/10.15283/ijsc21005.

\section{References}

1. Bubb KJ, Aubdool AA, Moyes AJ, Lewis S, Drayton JP, Tang O, Mehta V, Zachary IC, Abraham DJ, Tsui J, Hobbs AJ. Endothelial C-type natriuretic peptide is a critical regulator of angiogenesis and vascular remodeling. Circulation 2019;139:1612-1628
2. Oka T, Akazawa H, Naito AT, Komuro I. Angiogenesis and cardiac hypertrophy: maintenance of cardiac function and causative roles in heart failure. Circ Res 2014;114:565-571

3. Boström P, Wu J, Jedrychowski MP, Korde A, Ye L, Lo JC, Rasbach KA, Boström EA, Choi JH, Long JZ, Kajimura $\mathrm{S}$, Zingaretti MC, Vind BF, Tu H, Cinti S, Højlund K, Gygi SP, Spiegelman BM. A PGC1- $\alpha$-dependent myokine that drives brown-fat-like development of white fat and thermogenesis. Nature 2012;481:463-468

4. Tachibana A, Santoso MR, Mahmoudi M, Shukla P, Wang L, Bennett M, Goldstone AB, Wang M, Fukushi M, Ebert $\mathrm{AD}$, Woo YJ, Rulifson E, Yang PC. Paracrine effects of the pluripotent stem cell-derived cardiac myocytes salvage the injured myocardium. Circ Res 2017;121:e22-e36

5. Wang H, Zhao YT, Zhang S, Dubielecka PM, Du J, Yano $\mathrm{N}$, Chin YE, Zhuang S, Qin G, Zhao TC. Irisin plays a pivotal role to protect the heart against ischemia and reperfusion injury. J Cell Physiol 2017;232:3775-3785

6. Zhang X, Hu C, Kong CY, Song P, Wu HM, Xu SC, Yuan YP, Deng W, Ma ZG, Tang QZ. FNDC5 alleviates oxidative stress and cardiomyocyte apoptosis in doxorubicin-induced cardiotoxicity via activating AKT. Cell Death Differ 2020;27:540-555

7. Yu Q, Kou W, Xu X, Zhou S, Luan P, Xu X, Li H, Zhuang J, Wang J, Zhao Y, Xu Y, Peng W. FNDC5/Irisin inhibits pathological cardiac hypertrophy. Clin Sci (Lond) 2019;133: 611-627

8. Li RL, Wu SS, Wu Y, Wang XX, Chen HY, Xin JJ, Li H, Lan J, Xue KY, Li X, Zhuo CL, Cai YY, He JH, Zhang HY, Tang CS, Wang W, Jiang W. Irisin alleviates pressure overload-induced cardiac hypertrophy by inducing protective autophagy via mTOR-independent activation of the AMPK-ULK1 pathway. J Mol Cell Cardiol 2018;121:242255

9. Wu F, Song H, Zhang Y, Zhang Y, Mu Q, Jiang M, Wang F, Zhang W, Li L, Li H, Wang Y, Zhang M, Li S, Yang L, Meng Y, Tang D. Irisin induces angiogenesis in human umbilical vein endothelial cells in vitro and in zebrafish embryos in vivo via activation of the ERK signaling pathway. PLoS One 2015;10:e134662

10. Liao Q, Qu S, Tang LX, Li LP, He DF, Zeng CY, Wang WE. Irisin exerts a therapeutic effect against myocardial infarction via promoting angiogenesis. Acta Pharmacol Sin 2019;40:1314-1321

11. Yang F, Wu R, Jiang Z, Chen J, Nan J, Su S, Zhang N, Wang C, Zhao J, Ni C, Wang Y, Hu W, Zeng Z, Zhu K, Liu X, Hu X, Zhu W, Yu H, Huang J, Wang J. Leptin increases mitochondrial OPA1 via GSK3-mediated OMA1 ubiquitination to enhance therapeutic effects of mesenchymal stem cell transplantation. Cell Death Dis 2018;9: 556

12. Zhang N, Ye F, Zhu W, Hu D, Xiao C, Nan J, Su S, Wang Y, Liu M, Gao K, Hu X, Chen J, Yu H, Xie X, Wang J. Cardiac ankyrin repeat protein attenuates cardiomyocyte apoptosis by upregulation of Bcl-2 expression. Biochim Biophys Acta 2016;1863:3040-3049 
13. Golpanian S, Wolf A, Hatzistergos KE, Hare JM. Rebuilding the damaged heart: mesenchymal stem cells, cell-based therapy, and engineered heart tissue. Physiol Rev 2016;96:1127-1168

14. Ranganath SH, Levy O, Inamdar MS, Karp JM. Harnessing the mesenchymal stem cell secretome for the treatment of cardiovascular disease. Cell Stem Cell 2012;10:244-258

15. Hsiao ST, Asgari A, Lokmic Z, Sinclair R, Dusting GJ, Lim SY, Dilley RJ. Comparative analysis of paracrine factor expression in human adult mesenchymal stem cells derived from bone marrow, adipose, and dermal tissue. Stem Cells Dev 2012;21:2189-2203

16. Deng J, Zhang N, Wang Y, Yang C, Wang Y, Xin C, Zhao J, Jin Z, Cao F, Zhang Z. FNDC5/irisin improves the therapeutic efficacy of bone marrow-derived mesenchymal stem cells for myocardial infarction. Stem Cell Res Ther 2020; 11:228

17. Mahajan UB, Chandrayan G, Patil CR, Arya DS, Suchal K, Agrawal Y, Ojha S, Goyal SN. Eplerenone attenuates myocardial infarction in diabetic rats via modulation of the PI3K-Akt pathway and phosphorylation of GSK-3 $\beta$. Am J Transl Res 2018;10:2810-2821

18. Wollert KC, Drexler H. Cell therapy for the treatment of coronary heart disease: a critical appraisal. Nat Rev Cardiol 2010;7:204-215

19. Parekkadan B, Milwid JM. Mesenchymal stem cells as therapeutics. Annu Rev Biomed Eng 2010;12:87-117

20. Park J, Lee JH, Yoon BS, Jun EK, Lee G, Kim IY, You $\mathrm{S}$. Additive effect of bFGF and selenium on expansion and paracrine action of human amniotic fluid-derived mesenchymal stem cells. Stem Cell Res Ther 2018;9:293

21. Korta P, Pocheć E, Mazur-Biały A. Irisin as a multifunctional protein: implications for health and certain diseases. Medicina (Kaunas) 2019;55:485

22. Lourenco MV, Frozza RL, de Freitas GB, Zhang H, Kincheski GC, Ribeiro FC, Gonçalves RA, Clarke JR, Beckman D, Staniszewski A, Berman H, Guerra LA, Forny-Germano L, Meier S, Wilcock DM, de Souza JM, Alves-Leon S, Prado VF, Prado MAM, Abisambra JF, Tovar-Moll F, Mattos P, Arancio O, Ferreira ST, De Felice FG. Exercise-linked FNDC5/irisin rescues synaptic plasticity and memory defects in Alzheimer's models. Nat Med 2019;25:165-175

23. Zhou X, Xu M, Bryant JL, Ma J, Xu X. Exercise-induced myokine FNDC5/irisin functions in cardiovascular protection and intracerebral retrieval of synaptic plasticity. Cell Biosci 2019;9:32

24. Tan Y, Ouyang H, Xiao X, Zhong J, Dong M. Irisin ameliorates septic cardiomyopathy via inhibiting DRP1-related mitochondrial fission and normalizing the JNK-LATS2 signaling pathway. Cell Stress Chaperones 2019;24:595-608

25. Colaianni G, Mongelli T, Colucci S, Cinti S, Grano M. Crosstalk between muscle and bone via the muscle-myokine Irisin. Curr Osteoporos Rep 2016;14:132-137

26. Grygiel-Górniak B, Puszczewicz M. A review on irisin, a new protagonist that mediates muscle-adipose-bone-neuron connectivity. Eur Rev Med Pharmacol Sci 2017;21:46874693

27. Lee CW, Hsiao WT, Lee OK. Mesenchymal stromal cell-based therapies reduce obesity and metabolic syndromes induced by a high-fat diet. Transl Res 2017;182: 61-74.e8

28. Yao D, Liu NN, Mo BW. Assessment of proliferation, migration and differentiation potentials of bone marrow mesenchymal stem cells labeling with silica-coated and aminemodified superparamagnetic iron oxide nanoparticles. Cytotechnology 2020;72:513-525

29. Ullah M, Liu DD, Thakor AS. Mesenchymal stromal cell homing: mechanisms and strategies for improvement. iScience 2019;15:421-438

30. Rabiee F, Lachinani L, Ghaedi S, Nasr-Esfahani MH, Megraw TL, Ghaedi K. New insights into the cellular activities of Fndc5/Irisin and its signaling pathways. Cell Biosci 2020;10:51

31. Ersahin T, Tuncbag N, Cetin-Atalay R. The PI3K/AKT/ mTOR interactive pathway. Mol Biosyst 2015;11:1946-1954

32. Lanza Cariccio V, Scionti D, Raffa A, Iori R, Pollastro F, Diomede F, Bramanti P, Trubiani O, Mazzon E. Treatment of periodontal ligament stem cells with MOR and CBD promotes cell survival and neuronal differentiation via the PI3K/Akt/mTOR pathway. Int J Mol Sci 2018;19:2341

33. Li JY, Ren KK, Zhang WJ, Xiao L, Wu HY, Liu QY, Ding T, Zhang XC, Nie WJ, Ke Y, Deng KY, Liu QW, Xin HB. Human amniotic mesenchymal stem cells and their paracrine factors promote wound healing by inhibiting heat stress-induced skin cell apoptosis and enhancing their proliferation through activating PI3K/AKT signaling pathway. Stem Cell Res Ther 2019;10:247

34. Deng J, Bai X, Feng X, Ni J, Beretov J, Graham P, Li Y. Inhibition of $\mathrm{PI} 3 \mathrm{~K} / \mathrm{Akt} / \mathrm{mTOR}$ signaling pathway alleviates ovarian cancer chemoresistance through reversing epithelial-mesenchymal transition and decreasing cancer stem cell marker expression. BMC Cancer 2019;19:618

35. Zhang D, Zhang P, Li L, Tang N, Huang F, Kong X, Tan $\mathrm{X}$, Shi G. Irisin functions to inhibit malignant growth of human pancreatic cancer cells via downregulation of the PI3K/AKT signaling pathway. Onco Targets Ther 2019;12: 7243-7249

36. Liu J, Huang Y, Liu Y, Chen Y. Irisin enhances doxorubicin-induced cell apoptosis in pancreatic cancer by inhibiting the PI3K/AKT/NF- $\kappa$ B pathway. Med Sci Monit 2019;25:6085-6096

37. Shi G, Tang N, Qiu J, Zhang D, Huang F, Cheng Y, Ding $\mathrm{K}$, Li W, Zhang P, Tan X. Irisin stimulates cell proliferation and invasion by targeting the PI3K/AKT pathway in human hepatocellular carcinoma. Biochem Biophys Res Commun 2017;493:585-591

38. Liu TY, Shi CX, Gao R, Sun HJ, Xiong XQ, Ding L, Chen Q, Li YH, Wang JJ, Kang YM, Zhu GQ. Irisin inhibits hepatic gluconeogenesis and increases glycogen synthesis via the PI3K/Akt pathway in type 2 diabetic mice and hepatocytes. Clin Sci (Lond) 2015;129:839-850 
39. Bilanges B, Posor Y, Vanhaesebroeck B. PI3K isoforms in cell signalling and vesicle trafficking. Nat Rev Mol Cell Biol 2019;20:515-534
40. Cheng CF, Ku HC, Lin H. PGC-1 $\alpha$ as a pivotal factor in lipid and metabolic regulation. Int J Mol Sci 2018;19:3447 